\title{
Enquête
}

Archives de la revue Enquête

$4 \mid 1988$

Varia

\section{Le jeu et la loi}

La stratégie paradoxale du cinéma buñuélien

\section{Marie-Claude Taranger}

\section{(2) OpenEdition}

Journals

Édition électronique

URL : http://journals.openedition.org/enquete/63

DOI : $10.4000 /$ enquete. 63

ISSN : 1953-809X

Éditeur :

Cercom, Éditions Parenthèses

Édition imprimée

Date de publication : 2 juin 1988

\section{Référence électronique}

Marie-Claude Taranger, « Le jeu et la loi », Enquête [En ligne], 4 | 1988, mis en ligne le 27 juin 2013,

consulté le 20 avril 2019. URL : http://journals.openedition.org/enquete/63 ; DOI : 10.4000/enquete.63

Ce document a été généré automatiquement le 20 avril 2019. 


\title{
Le jeu et la loi
}

\author{
La stratégie paradoxale du cinéma buñuélien ${ }^{1}$
}

\author{
Marie-Claude Taranger
}

On sait que les sociétés déterminent leurs formes. L'art est soumis à des lois qui règlent son émergence, sa recevabilité, sa lisibilité. Cela est d'autant plus net en ce qui concerne le cinéma que le poids de l'industrie d'une part, de la technique d'autre part, y est certainement plus fort que dans les autres domaines, les normes étant de ce fait à la fois moins diverses et plus contraignantes. Dès lors, la production artistique en général et cinématographique en particulier peut-elle fonctionner autrement que ne l'imposent les lois, ou les coutumes, en vigueur? Peut-elle signifier autre chose que ce que permettent de signifier les cadres matériels et conceptuels hors desquels elle ne peut exister? Dans quelles conditions? À quel prix ? Avec quels effets? C'est à cet ensemble de questions que me semble permettre d'apporter des éléments de réponse l'étude de la production cinématographique de Luis Buñuel.

Une brève évocation du début de sa carrière peut montrer pourquoi.

3 L'œil tranché d'Un chien andalou², le scandale de L'âge d'or: images et faits sont connus de tous. C'est clairement sous le signe de la rupture que se place d'emblée le projet cinématographique de Buñuel. Vont donc de pair dans ces premiers films l'agression de la sensibilité, l'innovation formelle, le rejet violent des valeurs morales. Mon propos ici n'est pas de m'interroger sur les raisons de cette rupture, mais sur ses possibilités d'expression et sa portée.

4 Pour ces premiers films, les faits semblent clairs. L'originalité du propos est autorisée par la marginalité de la production (recours au mécénat, diffusion " parallèle »). Elle n'en est pas moins radicalement sanctionnée par les institutions gardiennes de l'ordre social mis en cause: manifestations, campagnes de presse et - pour finir - interdiction totale répondent à la provocation de L'âge d'or.

Rien que de fort simple dans ce prologue. Les questions portent sur la suite, et d'abord sur le fait même qu'il puisse y avoir une suite. Buñuel aurait pu, aurait dû, être le Rimbaud du cinéma. On peut considérer qu'il l'a été jusqu'à la fin des années 1940. Au total cependant, 
il compte parmi les cinéastes dont la carrière a été la plus riche et la plus brillante. Comment s'est donc résolue la contradiction initiale de son projet aux normes cinématographiques et sociales? Mon hypothèse est qu'elle ne s'est pas résolue, mais que le cinéma de Buñuel est parvenu à élaborer une stratégie efficace et subtile qui lui permette de placer cette contradiction au cœur des films, au lieu qu'elle en compromette l'existence.

\section{Le principe du jeu}

6 Le principe de cette stratégie est simple, même si son application est difficile et périlleuse : il s'agit d'entrer dans le jeu, mais d'en détourner la règle et de la jouer contre elle-même jusqu'à en mettre en cause les fondements mêmes.

Deux exemples, empruntés à l'un des derniers films de Buñuel, Le charme discret de la bourgeoisie, peuvent servir à donner sans tarder une idée précise de ce que recouvre cette stratégie.

Imaginez une femme mariée rendant visite à son amant. Ils viennent de passer dans la chambre à coucher quand on sonne à la porte : c'est le mari trompé. Ajoutez à cette situation de vaudeville les ingrédients habituels du film «commercial»: mise en scène "propre », à la fois claire et habile, acteurs connus (ici Delphine Seyrig et Fernando Rey par exemple) : c'est la première phase du jeu buñuélien, l'apparence d'une conformité totale.

9 Que se passe-t-il alors? Dans l'histoire, rien. De tous les développements possibles de la situation initiale, aucun n'est retenu par le récit: l'amant ouvre la porte, le mari rencontre sa femme, mais l'adultère n'est cependant ni caché ni découvert, l'époux n'apparaît ni offensé, ni indulgent, ni complice. Sans cesser d'être présente, la situation initiale semble totalement évacuée: le seul résultat de cette scène est dérisoire et mondain - la transmission d'une invitation à dîner. C'est la deuxième phase du jeu : le déroulement du film nie les relations de contradiction sur lequel il s'est d'abord construit. Épouse infidèle et mari trompé sont là, mais ils sont littéralement insignifiants. Au lieu que l'exploitation dramatique de l'infraction renvoie à l'ordre troublé, cet ordre est plus qu'attaqué : il est totalement récusé ; il n'est pas détruit : il n'existe pas.

Second exemple, toujours emprunté au même film : l'utilisation du rêve.

11 Les aventures que raconte Le charme discret de la bourgeoisie se partagent, d'une façon très buñuélienne et cependant assez banale, entre le rêve et la réalité. L'opposition semble d'abord explicite et claire. Les indications du dialogue sont confirmées par des oppositions thématiques et stylistiques: les rêves sont pleins de fantômes et de fantasmes, obscurs, étranges, d'une incohérence très freudienne. Mais, de plus en plus, le système de répartition se dérègle : malgré la persistante mais illusoire clarté des réveils soudains, les deux mondes échangent peu à peu leurs traits jusqu'à rendre finalement leur frontière indécidable. Structure paradoxale : alors qu'à petite échelle les critères de séparation semblent toujours pouvoir fonctionner, sur l'ensemble du film pourtant la répartition s'avère définitivement impossible. Alors que le rôle usuel des règles est d'autoriser un classement, Buñuel les emploie de manière à interdire le classement. Ainsi se trouve mise en cause une certaine représentation des rapports entre "rêve " et " réalité » (entre "vrai » et "faux »), et par là un des grands principes qui nous sert traditionnellement à ordonner monde et discours. Ainsi s'articule un discours impossible. 


\section{La contradiction des réceptions} aussi les récompenses officielles. Palme d'or au festival de Cannes en 1950 pour Los Olvidados, en 1961 pour Viridiana ; prix spécial du jury en 1959 pour Nazarin. Grand prix au festival de Sestri-Levante en 1962 pour L'ange exterminateur. Ours d'or au festival de Berlin en 1966 pour Belle de jour. Coquille d'or à Saint-Jacques-de-Compostelle en 1977 pour Cet obscur objet du désir. Etc.

D'un côté, la conformité se trouve sanctionnée par la reconnaissance sous toutes ses formes. Il y a d'abord le succès commercial. À partir des années 1950, la réussite de Et la consécration de toutes parts. En France, en 1974, le Premier ministre inclut la projection du « dernier Buñuel » dans le programme d'un voyage officiel. À Hollywood, en 1972, Georges Cukor organise à l'occasion du passage de Buñuel dans la ville un repas de consécration qui réunit à ses côtés tous les « grands » du cinéma, de John Ford à William Wyler, de Billy Wilder à Alfred Hitchcock. En 1981, le festival de Cannes fête les quatrevingts ans de Buñuel en organisant un «hommage", aussitôt repris par la télévision. Buñuel publie ses mémoires... Enfin, en juillet-août 1983, c'est le chœur unanime des oraisons funèbres. Le même Figaro qui avait réclamé l'interdiction de L'âge d'or titre sur double page : «Mort d'un grand d'Espagne ».

D'un autre côté cependant, scandales et censures sanctionnent de façon persistante la poursuite du dérèglement. Scandale de Viridiana en 1961. Le régime franquiste commence par fêter l'enfant prodigue qui le représente au festival de Cannes; mais au moment où le film est primé, l'Espagne le désavoue et finalement l'interdit. Interventions répétées de la censure : pour Viridiana, pour Belle de jour, pour Le charme discret de la bourgeoisie.

Sans parler des désaccords des producteurs (Oscar Dancigers pour Los Olvidados ou les frères Hakim pour Belle de jour par exemple), ou des diverses formes de refus des collaborateurs : la coiffeuse de Los Olvidados quittant le film parce qu'en désaccord avec l'image qu'il donne de la mère, l'interprète d'El bruto refusant de dire certaines répliques, l'opérateur de Nazarin sommé d'orienter sa caméra à l'opposé de ce qu'il avait d'abord prévu, le monteur de L'ange exterminateur catastrophé de ce qu'il prenait pour de grossières erreurs de montage.

Formes condensées de ces réceptions contradictoires : les controverses autour des films. Le débat sur Nazarin est certainement le plus célèbre : le rapport du personnage au Christ et du film au christianisme divisa spectateurs et critiques. Mais ce débat n'est par le dernier : en 1974 par exemple, Le fantôme de la liberté donna lieu aussi à une controverse vive, bien que formulée en d'autres termes (elle portait cette fois précisément sur l'appréciation du conformisme de Buñuel et de la portée critique du film).

Un terrain privilégié pour saisir à travers ses effets le caractère contradictoire de la stratégie buñuélienne est le terrain de la signification. Reprenant les formes habituelles au cinéma, les films promettent un sens dont leur dérèglement perturbe l'émergence. De là divers types de réactions. Certaines sont des sanctions directes de l'écart. Le rire par 
exemple, particulièrement attesté pour les derniers films (« On rit. On a raison de rire », dit un critique). Ou, plus fréquente, la perplexité.

Tournons-nous du côté des spectateurs, et des critiques qui en sont à certains égards les relais. Les spectateurs sont intrigués. Ils interrogent : qu'y a-t-il dans la mystérieuse boîte que le gros client coréen fait écouter à Belle de jour? Et dans le sac que transporte partout le héros de Cet obscur objet du désir? Que font les personnages du Charme discret de la bourgeoisie sur la route déserte où on les voit à plusieurs reprises ? Pourquoi l'irruption d'un ours dans la somptueuse demeure de L'ange exterminateur?

Les critiques refusent d'expliquer. Et expliquent. Il n'y a rien à comprendre. Les amis du Charme discret marchent à pied parce que leur classe est condamnée à l'errance, parce que Buñuel a rêvé de route, parce que leur voiture est en panne. L'ours de L'ange exterminateur, c'est l'inconscient, l'animalité toujours menaçante, l'URSS, etc.

Le leitmotiv qui exprime à la fois l'interrogation et la dénégation, c'est le «symbole » - tarte-à-la-crème de la littérature buñuélienne. Deux textes sur trois (ou neuf sur dix ?), deux interviews sur trois (ou neuf sur dix ?) parlent de symbole. En général pour en récuser la pertinence: "Il n'y a pas de symbole ", «Il n'y a rien de symbolique ». La dénégation ici importe moins que sa nécessité. Si l'interprétation symbolique a sans cesse besoin d'être écartée, c'est parce qu'elle menace sans cesse : réponse providentielle aux énigmes des films, elle en souligne l'existence avec insistance. Elle signale, tout au long du cinéma buñuélien, la permanence de zones d'opacité : ici, on cesse de comprendre, ici, les repères se perdent, les outils qui servent d'ordinaire à construire le sens ne sont plus d'aucune utilité.

Faillite de la transparence qui renvoie à l'œil tranché et au scandale : l'illisibilité du film ou la blessure du regard sont deux versants d'une même rupture, dont l'incompréhension courante ou le rejet violent sont deux des sanctions possibles.

Dans la construction de la signification, une autre réaction possible à la contradiction buñuélienne est sa résolution. L'anomalie doit à son caractère d'anomalie d'être corrigée ou, ce qui revient au même, de ne pas être perçue. Divers procédés apparaissent ainsi dans les commentaires pour rétablir l'ordre que les films bouleversent.

Normalisation par omission: on ne mentionne pas l'infraction ou l'étrangeté. Par exemple, la plupart des récits du Charme discret de la bourgeoisie évoquent la scène d'adultère que j'ai rappelée ci-dessus sans rien dire de sa déroutante absence de suites.

Normalisation par addition: on résout les contradictions par l'adjonction de termes médiateurs. Aux spectateurs désorientés par la revendication paradoxale des manifestants du Fantôme de la liberté, qui se révoltent aux cris d'« À bas la liberté », on explique : «À bas la fausse liberté », ou, plus savamment : «À bas la liberté, si c'est la liberté des codes, et non des corps $»^{3}$.

27 Remise à l'endroit de ce que les films mettent à l'envers: quand le récit buñuélien intervertit la cause et la conséquence, comme dans l'histoire de l'évêque-jardinier du Charme discret de la bourgeoisie, on corrige pour raconter «normalement » : " un évêque peu ordinaire" «se camoufle sous le tablier d'un jardinier" "pour échapper aux poursuites ".

28 Consciente ou inconsciente, la censure des spectateurs et des commentateurs prolonge en aval du film la censure exercée en amont par les institutions publiques et privées. Un exemple éclatant concerne le rapport du rêve et de la réalité dans Belle de jour où, comme 
dans Le charme discret de la bourgeoisie, l'aberration savante qui préside à la répartition des indices de réalité empêche finalement le partage entre rêve et réalité: clairement distinguées au départ, les séquences "réelles» et les séquences fantasmatiques échangent en effet leurs caractéristiques thématiques et stylistiques de façon de plus en plus complexe et inextricable. Néanmoins, nombre de spectateurs et de commentaires manipulent ces indices pour rétablir la possibilité du partage et permettre, par exemple, de raconter le film. De même fit l'exploitant (ou le distributeur) qui, sans nuance, amputa un jour une des copies du terme définitivement contradictoire que constitue l'avantdernière séquence. Alors que l'histoire s'achève sur deux dénouements incompatibles que rien ne permet d'articuler avec certitude (1. Le mari de l'héroïne est complètement paralysé. 2. Il se lève de sa chaise d'infirme et propose un séjour aux sports d'hiver), j'ai vu un jour le film avec une seule des deux fins (la première). Et c'était bien sûr beaucoup plus simple!

On peut dire au total qu'à la stratégie contradictoire que Buñuel élabore et met en œuvre dans ses films répond une réception elle-même contradictoire, et de ce fait instable, partagée entre l'éloge et le rejet, l'adhésion et la perplexité, la compréhension et l'incompréhension, les attitudes contradictoires se juxtaposant sans s'articuler, souvent au sein des mêmes commentaires. Nombreux sont ainsi les critiques du Charme discret de la bourgeoisie qui, tout en affirmant que le film mêle jusqu'à les rendre indistincts le "vrai » et le «faux », font d'autre part comme s'il n'en était rien: alors même que les séquences de "rêves » ne peuvent être clairement délimitées, on ne renonce ni à les caractériser ni à les expliquer. Ainsi la contradiction des films se traduit-elle et se poursuit-elle par les contradictions qu'ils suscitent. Ces contradictions dans la réception peuvent servir de repères pour l'analyse.

\section{Applications}

Les principes sont clairs.

Reste maintenant l'essentiel, c'est-à-dire la description de leur application. S'agissant d'une pratique paradoxale, qui a pour effet de déplacer les bornes du possible, le constat des faits est essentiel. D'autant que la réussite d'une entreprise de ce type est évidemment difficile: fondée sur la déception, elle se doit, pour être efficace, de la renouveler constamment. Il faut que, de film en film, de séquence en séquence, producteurs et spectateurs restent dans le jeu. Il faut que la règle soit suffisamment respectée pour que son infraction conserve un sens, que donc l'ordre se dessine et se redessine pour être continûment mis en cause. Comment cet équilibre peut-il être maintenu sans que l'un des termes de la contradiction l'emporte sur l'autre?

Il ne peut s'agir ici de donner à cette question une réponse qui prétende aussi peu que ce soit à l'exhaustivité. Tout au plus est-il possible de donner quelques points de repère sur les procédures, employées et l'extension de leurs applications. Pour cela, je me référerai surtout, pour sa proximité et pour son exemplarité, à la dernière partie de la production buñuélienne, et notamment au triptyque que constituent La voie lactée, Le charme discret de la bourgeoisie et Le fantôme de la liberté. 


\section{Le charme discret de l'excellence}

Pour ce renouvellement constant de l'équilibre, il faut souligner d'abord combien sont essentiels les éléments de conformité aux normes les plus courantes de la production cinématographique, même ceux que pourrait masquer leur trop grande évidence.

La normalité de Buñuel a plusieurs aspects.

Elle est d'abord conformité fondamentale aux habitudes de représentation et de narration que le cinéma a empruntées aux traditions picturales et romanesques. Repris le choix le plus banal d'un cinéma "représentatif-narratif » (Buñuel n'est ni MacLaren ni Warhol), les cadres qui fournissent d'ordinaire leur armature aux narrations cinématographiques sont ensuite très efficacement mis en place. Le pacte narratif est en général très clairement celui de la "fiction"; les éléments classiques du récit sont définis de façon usuelle; ainsi les temps, les lieux, les actions, et surtout les personnages: ils sont précisément dessinés par les scénarios qui leur attribuent classiquement des caractéristiques psychologiques et sociales: ambassadeurs ou préfets, militaires ou médecins, courtois ou grossiers, braves ou craintifs, ils ont leurs façons d'être propres en même temps que leur cohérence sociale; ils respectent d'autre part une hiérarchie traditionnelle qui les répartit clairement entre personnages principaux et secondaires, « héros » et « comparses ».

Au-delà de cette conformité fondamentale, la conformité aux normes est aussi conformité conjoncturelle: durée standard, technique standard, «casting» standard, les films de Buñuel suivent normalement l'évolution des formes, des techniques et des modes. Tandis que, par exemple, la couleur s'installe à partir de Belle de jour, la célébrité des noms et des visages intègre sans conteste les films dans le fonctionnement le plus courant du cinéma : au fil du temps, on y retrouve, comme ailleurs, Georges Marchal, Michel Piccoli, Jeanne Moreau, Catherine Deneuve, et bien d'autres.

Cette conformité d'autre part est magistrale et prend souvent des allures d'excellence. L'écriture filmique proprement dite par exemple met en œuvre à la perfection les principes d'unité, de hiérarchie, de transparence et de redondance qui régissent le découpage classique. On peut parler sans faillir de "transparence cristalline » et de "classicisme hollywoodien". De même pour la mise en scène au sens propre: la perfection dans le choix et la direction d'acteurs est un des aspects des films toujours soulignés. On dit par exemple de Delphine Seyrig dans Le charme discret de la bourgeoisie: « Il faut l'entendre moduler des phrases telles que "Ça sent le vol-au-vent financière". On croirait qu'elle a dit cela tous les jours, toute sa vie."

Ainsi les œuvres, en dépit de ce qui peut constituer d'autre part leur originalité, multiplient-elles les références au patrimoine culturel et aux pratiques artistiques de la société qui les produit, et souvent aux plus reconnues. Omniprésente, la référence au récit romanesque est notable malgré sa banalité, à cause de sa banalité ; Lewis, Emily Brontë, Galdos, Becquer, Pierre Louÿs, Octave Mirbeau, Joseph Kessel, et d'autres : Buñuel, comme beaucoup de cinéastes, adapte les auteurs les plus divers; sur ce terrain commun, il retrouve du reste des réalisateurs aussi divers que Renoir ou Wyler, Sternberg ou Duvivier, qui ont, comme lui, adapté Le journal d'une femme de chambre, Les hauts de HurleVent ou La femme et le pantin. 

l'opéra (Wagner ou La Tosca), au boulevard (Feydeau par exemple), à l'Ancien et au Nouveau Testament, à la tradition catholique ( $\mathrm{La}$ légende dorée), à la littérature théologique, au marquis de Sade. L'essentiel ici n'est pas le détail, mais le nombre et l'ampleur des références : par là, comme par l'emploi des techniques habituelles et des vedettes consacrées, le cinéma buñuélien s'articule constamment sur un contexte artistique et culturel auquel il reprend ses formes et sa matière. subversion.

\section{Contes et mécomptes}

41 De cette subversion, une première caractéristique est la généralité : une fois les cadres mis en place, il n'est guère d'aspects des films qu'elle épargne. Même les éléments les plus conformes peuvent être subtilement dévoyés: dans la clarté du découpage, des aberrations passagères créent de presque imperceptibles failles; dans le jeu des acteurs, une emphase et un soulignement systématiques tendent à transformer la perfection en excès.

Une autre caractéristique est la réflexivité : le discours buñuélien est lui-même l'un des objets de la mise en cause qu'il opère.

La conduite du récit peut constituer un exemple qui permette de mettre en évidence cette double caractérisation et d'en préciser les enjeux.

Le bouleversement de l'anecdote est souvent très manifeste dans les films de Buñuel, auxquels il donne une apparence "surréaliste " immédiate et un aspect de "cadavres exquis ». À l'origine de ce bouleversement se trouvent des principes simples dont l'application aboutit à modifier l'économie attendue de la narration, et par là à en mettre en cause les fondements. Les deux premiers principes sont des principes symétriques de multiplication et de suppression des éléments du récit; le troisième est un principe d'altération du schéma narratif.

Le principe de multiplication est susceptible d'affecter également tous les constituants du récit. Profusion des lieux et des temps dans La voie lactée, dont le récit éclate entre de multiples époques et de multiples régions. Profusion plus générale des personnages, des histoires, des genres. La multiplication des " héros » est très nette dans Le charme discret de la bourgeoisie qui place au cœur de son récit un groupe de six personnages auxquels il convient d'ajouter tous ceux qui viennent les rejoindre ou les remplacer au-devant de la scène et du récit : évêque jardinier, lieutenant meurtrier de son père, sergent amoureux de sa mère, «brigadier sanglant ", etc. Multiplication plus nette encore dans Le fantôme de la liberté qui propose au moins sept récits successifs organisés chacun autour de personnages différents. Multiplication que redouble une multiplication plus grande encore des histoires racontées et des genres auxquels elles peuvent se rattacher : on passe de la « comédie psychologique » à l'aventure policière, du réalisme au fantastique, du rire au drame.

Que cette prolifération fasse violence aux habitudes du récit filmique, le plus souvent construit sur un « héros » qui constitue le centre permanent d'une histoire unique, et du spectateur, accoutumé à s'appuyer sur cette permanence, un indice peut être la constance 
avec laquelle la critique souligne le renouvellement des personnages du Fantôme de la liberté, et les termes employés pour cela: on parle de la «frustration» de spectateurs « déconcertés » quand le film « abandonne » ses héros. Autre indice non négligeable : les génériques des films. Comme la traditionnelle mention des interprètes par la critique ou la publicité, ils ont un aspect d'énumération relativement inhabituel : là où suffisent d'ordinaire un ou deux noms écrits en gros, on ne peut échapper ici à la liste (" par ordre alphabétique » ou "d'apparition à l'écran »).

Cette prolifération de la matière narrative doit l'essentiel de sa portée au fait qu'elle se combine avec un principe opposé de suppression des éléments du récit attendu. Les exemples les plus spectaculaires de telles suppressions sont liés aux interruptions soudaines dont Buñuel est coutumier. Ainsi le réveil des dormeurs du Charme discret de la bourgeoisie interrompt-il les récits que constituent leurs rêves, supprimant du même coup suites et dénouements : le geste du «brigadier sanglant » ouvrant les portes des prisons reste sans conséquence au réveil du commissaire qui le rêve, puisqu'il n'y a dans les faits ni fantôme ni libération.

Le fantôme de la liberté aboutit aux mêmes effets en faisant bifurquer brutalement son récit sur des personnages de rencontre ou d'autres branches de l'histoire. Ainsi, au moment où le médecin s'apprête à lire la lettre qu'un facteur a remise en rêve à son client (sic), son assistante l'interrompt: "Nous ne connaîtrons donc jamais le contenu de la lettre ", regrette la critique. Ainsi, au moment où la lecture d'un procès-verbal va permettre d'expliquer par quel mystère a pu être «retrouvée» une petite fille qui n'avait pas disparu, le lecteur part brusquement pour un rendez-vous qu'il avait oublié : là non plus, le spectateur ne saura jamais le fin mot de l'histoire.

50 Ces interruptions très manifestes ne sont cependant que les applications les plus visibles d'un procédé constamment employé dans les films : les silences sont parfois plus discrets (c'est souvent le cas dans La voie lactée); les événements peuvent non seulement ne pas être racontés, mais encore manquer à se produire (ainsi après la découverte de l'adultère $\mathrm{du}$ Charme discret de la bourgeoisie). Ce sont tous les maillons de la chaîne narrative qui peuvent être tour à tour subtilisés : aux causes sans conséquences dont on vient de citer des exemples s'ajoutent des effets sans causes, et des développements absents.

51 Effets sans causes: on peut ici prendre pour exemples toutes les situations mystérieuses de La voie lactée. "D'où est sorti », comme le demande un personnage, le nain qu'on voit tout à coup marcher aux côtés du "Père »? Pourquoi le prêtre de l'auberge espagnole revient-il nuitamment parler aux occupants des chambres en cachant un sabre sous sa soutane?

52 L'absence des développements attendus malgré la présence canonique de débuts et de fins est, quant à elle, très manifeste dans Le charme discret de la bourgeoisie. De l'histoire de trafic de drogue qu'il semble à certains égards raconter, il ne laisse apparaître que quelques bribes, supprimant tous les maillons intermédiaires entre une présentation initiale banale (scène de livraison de "marchandise») et des dénouements brutaux (arrestation par la police, exécution par une bande rivale). Autre cas exemplaire dans le même film, variante du même procédé, avec les bruits de fond divers mais répétés qui empêchent le spectateur de saisir la teneur des propos quand ministre et commissaire expliquent qu'il faut libérer les trafiquants de drogue, ou quand une jeune terroriste développe ses arguments révolutionnaires. 

le dérèglement profond des récits vient de ce qu'ils sont à la fois pléthoriques et lacunaires. Si leur caractère lacunaire les distingue des types de narration où la profusion ne compromet pas la cohérence (le film «à sketches » par exemple), leur caractère prolifique les distingue aussi des récits elliptiques, où les manques peuvent définir une autre esthétique, sans pour autant détruire le récit même.

Aux effets perturbateurs de ces deux principes s'ajoutent ceux d'un troisième principe, qui est un principe d'altération du schéma narratif, et dont les formes sont diverses. Substitution à un élément attendu d'un élément déplacé, voire contradictoire ; ainsi, dans Le fantôme de la liberté, lorsque des cartes postales qui provoquent le scandale se révèlent représenter l'Arc de Triomphe et la basilique du Sacré-Cœur. Ou encore permutation de termes à l'intérieur d'une situation ou d'une séquence d'événements : ainsi, toujours dans Le fantôme de la liberté, on défèque en compagnie et on mange dans le secret des cabinets particuliers. Ici encore, les infractions les plus spectaculaires voisinent avec les dérèglements les plus ténus, tel le subtil désordre introduit dans les références théologiques de La voie lactée, lorsqu'une proposition orthodoxe se glisse dans une énumération d'hérésies.

Inversions, gauchissements, aberrations: Buñuel associe constamment des objets, des faits, des circonstances qui d'ordinaire sont dissociés ou s'excluent. "N'importe quoi ", dit-on, « peut faire irruption dans un film de Buñuel ». N'importe quel attribut peut aller avec n'importe quel prédicat : il y a des «évêques-jardiniers » et des «tueurs-poètes ». N'importe quelle action peut être le fait de n'importe quel sujet: les gendarmes chahutent, les soldats racontent leurs rêves, les maitres d'hôtel dissertent sur la double nature du Christ.

ces principes qui portent le dérèglement à tous les niveaux de la narration, la définition correspond bien à ce qui a été dit d'emblée de la stratégie buñuélienne. Car il s'agit de bouleversements syntaxiques; la matière sur laquelle ils s'exercent peut donc rester familière, voire banale (et c'est ce qui précisément permet au spectateur d'entrer dans le jeu), la différence et l'anomalie venant des agencements.

Aucun de ces principes cependant - et encore moins telle ou telle de leurs applications particulières - ne pourrait suffire pour décrire et expliquer le dérèglement spécifique de la narration buñuélienne.

En effet, les discours artistiques en général et les films et leurs récits en particulier se construisent couramment sur des écarts et des infractions à la fois parce que l'infraction est à la base même de la dynamique du récit, et parce que l'art se caractérise par la recherche de l'originalité (aussi les principes de dérèglement que j'ai repérés sont-ils également au cœur de la rhétorique).

59 Ce qui fait donc la spécificité de l'infraction buñuélienne, c'est sa généralité. C'est le fait que ces principes de dérèglement conjuguent leurs effets, et cela à tous les niveaux du récit. La multiplicité des ruptures et leur imbrication éloignent ces films non seulement d'un archétype de narration " classique », mais aussi bien de modèles substitutifs, comme les genres comique ou fantastique, ou encore des recherches du " cinéma d'auteur ", qui peuvent modifier partiellement les agencements simples et attendus, sans cesser pour autant d'être à la fois reconnus et recevables.

Quelques exemples peuvent permettre de préciser ce propos. 


\section{Infractions et récits}

61 Le charme discret de la bourgeoisie met en œuvre un type de dérèglement spécifique, qui consiste à brouiller un scénario policier par des événements banalement empruntés à la sphère de la "vie quotidienne ", très souvent représentée par la référence à la nourriture. Par exemple, alors que les héros du film viennent d'être criblés de balles et baignent dans leur sang, le seul survivant, qui s'était caché sous la table, trahit sa présence en essayant d'attraper une tranche de gigot dans une assiette. Brouillage comparable: les bourgeois trafiquants de drogue sont terrorisés en apprenant que leurs hôtes se sont enfuis par la gouttière et ils prennent la fuite à leur tour, craignant une dénonciation ou une rafle ; en fait, il ne s'agissait que d'une escapade derrière un buisson, motivée par un désir irrésistible et de savoureuses considérations de convenance.

Or, ce type de brouillage est un ressort tout à fait traditionnel du film comique.

63 Ainsi, dans un film comique contemporain du Charme discret de la bourgeoisie, Le grand blond avec une chaussure noire, toute une cascade d'effets résulte de la présence d'une banale rage de dents au milieu d'une affaire compliquée d'espionnage. Et l'anomalie qui donne son titre au film (le héros porte en effet deux chaussures de couleurs différentes) pourrait constituer un bon exemple d'altération narrative.

Mais si, dans ce cas, l'irruption de faits étranges et de motivations déplacées a bien pour effet de perturber le fonctionnement normal du film policier, c'est cette perturbation même qui devient alors le sujet du film. La tradition veut de surcroît que, dans ce type de situation, l'aventure sorte à la fois triomphante et renouvelée des périls qui l'ont menacée. Dans cette logique, la chaussure dépareillée du " grand blond» connaît donc une fortune étonnante et se trouve à l'origine d'une série de rebondissements spectaculaires.

Dans un cas de ce genre, il y a donc bien une perturbation du récit (en l'occurrence le scénario policier) mais limitée: le brouillage par le quotidien n'exclut ni les rebondissements romanesques ni une construction du récit au demeurant fort classique, autour d'un héros, d'une action principale (parodie d'action policière), sur laquelle se greffent des actions secondaires, dans un cadre homogène et un genre défini (le comique).

66 Rien de tel chez Buñuel : si les incongruités peuvent être du même ordre (par exemple avec le télescopage constant du trivial et du policier dans Le charme discret de la bourgeoisie), le brouillage «tombe à plat » comme le reste; il n'est à l'origine d'aucune péripétie, et les malentendus ou les bizarreries ne débouchent jamais sur aucun récit. Par exemple, lorsque, dans un salon de thé, les trois héroïnes apprennent qu'il n'y a ni thé, ni café, ni infusion, ni lait, ni cognac, l'invraisemblance un instant prometteuse de la coïncidence se brise aussitôt - et définitivement - sur l'explication «sans histoires » du garçon (une affluence exceptionnelle). Ce qui crée surtout l'étrangeté de ces situations dans le film, c'est justement qu'il se donne la peine de les reproduire et de les mettre en valeur (par l'accumulation, et les réactions à la fois des amies - qui s'étonnent - et du garçon - qui s'excuse avec insistance -), mais pour ne rien en faire. Au lieu donc que les étrangetés présentées alimentent un récit parodique mais efficace, elles constituent des butées sur lesquelles se brise au contraire la narration. 
On arriverait aux mêmes conclusions à partir d'une comparaison avec des films - ou d'autres récits - « fantastiques » ou de science-fiction.

Si on met par exemple en parallèle avec Le fantôme de la liberté, Alphaville, de Jean-Luc Godard, ou La convention Belzébir de Marcel Aymé ${ }^{4}$, nombre d'anomalies paraissent comparables : ici les monuments sont pornographiques, là la poésie est interdite et on exécute un homme parce qu'il a pleuré à la mort de sa femme (Alphaville); ici, le tueur est libéré triomphant, là, l'homicide est un sport de classe, à la fois licite et réglementé ( $L a$ convention Belzébir ). Cependant, chez Godard ou Marcel Aymé, si le bouleversement est flagrant, il a une certaine stabilité : une fois ces lois étranges établies, le récit fonctionne sur elles - et à partir d'elles - et fonctionne "normalement »: il y a des héros, une intrigue, une continuité... Dans Le fantôme de la liberté au contraire, les règles du monde changent à chaque instant, et ce ne sont pas seulement certaines normes de la représentation vraisemblable du monde, mais quasiment toutes les normes à la fois que le récit enfreint.

Et de quelque côté qu'on se tourne, le constat est le même. Les films " psychologiques », certes, n'exploitent guère les situations qu'ils présentent pour en tirer des rebondissements dramatiques, mais ils les utilisent pour " peindre » leurs personnages, là où trois films de Buñuel se contentent manifestement d'entasser des esquisses sans « fouiller » le moindre portrait.

Les « voyages à travers le temps » mélangent bien les époques et les contextes, mais ces mélanges obéissent à des lois et n'excluent pas, sur d'autres points, la cohérence du récit.

\section{La norme}

71 D'exemple en exemple, cette énumération peut conduire à préciser ce que recouvre la « norme » à laquelle je me réfère sans cesse pour décrire les bouleversements buñuéliens, et la façon dont elle peut être appréhendée.

La «norme ", ou encore l'« économie habituelle des données ", c'est l'ensemble des attentes du spectateur telles que façonnées par les autres films et les autres récits. Cet ensemble est certes mobile et variable: même si l'on choisit (ce que je fais), de situer exclusivement dans le contexte où les films ont été produits, tous les spectateurs n'ont pas les mêmes références. Il a cependant des caractéristiques assez marquées pour être saisissables : la place accordée aux vedettes est par exemple un fait très apparent.

73 Essayer d'appréhender cet ensemble directement et globalement me semble une tâche démesurée. Il me paraît possible en revanche d'en cerner certains aspects, à partir de comparaisons avec des exemples précis, qui ont valeur d'échantillons. Ce ne sont chaque fois que des échantillons, mais ces comparaisons limitées (avec tel ou tel exemple de film policier, comique, ou fantastique) me semblent devoir leur portée à la très grande redondance des productions cinématographiques : les films, et plus encore les films d'un genre, ont entre eux beaucoup de points communs; pour chaque trait, on peut citer un exemple mais on pourrait aussi en citer mille.

D'autre part, pour apprécier la stratégie buñuélienne, les diverses références possibles (narrations classiques, films comiques, films fantastiques, etc.) ont la même pertinence, faite de pertinence et d'impertinence à la fois. Pertinence, parce que de tous côtés les films de Buñuel exhibent leur parenté avec les films et les récits qu'on peut voir alentour. Impertinence parce que de ces divers « modèles » aux pratiques buñuéliennes, le décalage 
est toujours flagrant et la distance irréductible. Les horizons sur lesquels se détachent les films de Buñuel sont vastes et nombreux; l'essentiel est qu'aucune de ces multiples perspectives ne peut redonner aux films la cohérence et l'unité que perturbe leur jeu.

\section{Désordre et critique}

Particulièrement intéressante pour préciser la portée de l'entreprise buñuélienne peut être à cet égard la comparaison des films de Buñuel avec des films qui semblent avoir une thématique comparable de " critique sociale ».

Prenons, encore une fois, les exemples du Charme discret de la bourgeoisie et du Fantôme de la liberté. Parmi les anomalies qu'on y repère, revient toujours l'attribution à des notables et des puissants de comportements illégitimes: l'ambassadeur se sert de sa valise diplomatique pour un trafic de drogue, le colonel fume de la marijuana, les policiers torturent, l'évêque assassine ; s'accumulent d'autre part, de la part des bourgeois les plus respectables, voire des représentants de l'ordre, adultères, incestes, exhibitions, pratiques de nécrophilie, etc. Portraits d'un iconoclaste, dit-on volontiers, qui ébranle et secoue l'édifice de la bourgeoisie. Cependant, si la représentation de toutes ces infractions peut effectivement prendre valeur critique, c'est au bouleversement du récit qu'elle le doit. Car la présence d'adultères, de crimes, de policiers délinquants et de notables débauchés n'est pas en elle-même suffisante pour donner une valeur critique aux discours et aux films. Sans quoi ce serait la quasi-totalité des récits filmiques, même les plus conformistes, qui pourrait faire figure de "pamphlets", puisque adultères, assassinats et autres bavures sont la matière première de leur narration. Plus précisément même, on ne compte plus les récits policiers où le coupable est, comme l'ambassadeur du Charme discret de la bourgeoisie, un " citoyen au-dessus de tout soupçon ", ni les récits comiques qui se gaussent des policiers ou des gendarmes.

C'est donc ici qu'intervient la fonction même du récit. On sait qu'un récit se construit à partir d'un déséquilibre initial : une contradiction est posée dont la résolution - qui est précisément ce que raconte le récit - permet de retrouver un équilibre et un ordre ${ }^{5}$. Ainsi, le récit policier part-il d'une contradiction entre la loi et le crime, la comédie de mœurs la plus classique d'une contradiction entre l'adultère et le pacte conjugal.

Cette définition place à la source du récit la référence à l'ordre, qui permet de définir l'infraction comme telle et crée la nécessité de la narration. Et elle replace au terme du récit cette présentation de l'ordre. Ordre initial et ordre final peuvent être identiques ou différents : l'élimination des contradictions en effet peut se faire soit par suppression d'un des contradictoires (ainsi, le coupable puni ou pardonné, la faute « disparaît »), soit par suppression de la relation de contradiction (un nouvel ordre est établi). Le récit donc dit l'ordre du monde ; il dit aussi la conservation ou la transformation de cet ordre.

La plupart des récits filmiques disent le retour à l'ordre. L'infraction s'intègre dans un récit qui en organise la sanction si bien que, quelles que puissent être l'abondance ou la gravité des infractions, la pérennité de l'ordre du monde se trouve finalement (ré)affirmée. Dans l'exemple du récit policier, le cas le plus simple est celui où le coupable - ainsi le policier criminel - est reconnu et puni. Mais de la transgression au triomphe de la loi, les médiations peuvent aussi être plus subtiles: l'infraction peut apparaitre finalement négligeable, elle peut être minimisée par ses causes (cas fréquent dans le film comique) ou rachetée par une autre action. 

qui est des situations, il y a des rencontres étonnantes entre ces films et, par exemple, $L e$ fantôme de la liberté : ainsi, dans Le gendarme de Saint-Tropez, les gendarmes ont exactement les mêmes comportements infantiles d'écoliers chahuteurs et indisciplinés que la classe des gendarmes du Fantôme de la liberté. Mais les infractions de ce type, qui sont à la source du comique, ne compromettent l'ordre que provisoirement et. superficiellement. Il s'agit de broutilles, souvent commises avec les meilleures intentions du monde, et au demeurant rachetées par d'autres actions, où se réaffirme avec éclat la conformité du héros à son statut et à son devoir (ainsi quand « le gendarme de Saint-Tropez » arrête un horrible tueur).

frappe par contraste dans les récits buñuéliens et les distingue immédiatement des films "ordinaires", c'est l'absence de médiations et de corrections, et par voie de conséquence, l'absence du retour à l'ordre qu'elles permettent: du fait du bouleversement général de la narration, les infractions ne fonctionnent pas comme infractions, aucun récit ne venant finalement rendre son crédit à la loi.

Cest dire la portee critique de toutes les ruptures et de tous les manques qui empêchent de résorber les anomalies par le récit de leur sanction. Que le récit s'arrête, que le film sans cesse passe à autre chose, permet ainsi au meurtre du colonel ou du jardinier dans Le charme discret de la bourgeoisie, à l'inceste et à l'exhibition masochiste dans Le fantôme de la liberté, de rester sans conséquences. Ainsi, à l'inverse de ce qui se passe le plus souvent, la présentation des désordres ne débouche-t-elle pas sur la réaffirmation de l'ordre.

Mais il y a plus grave. Car, comme je l'ai déjà fait observer sur les premiers exemples, dans le brouillage du récit, l'ordre et le désordre cessent de se distinguer. Garder, comme le font les films de Buñuel, les données courantes du récit filmique, mais supprimer sans cesse le récit lui-même, revient à nier totalement le caractère contradictoire des éléments habituellement traités comme tels. Donc à nier l'ensemble des relations d'exclusion qui constituent l'«ordre» généralement pris comme référence. Donc à remettre fondamentalement en question la réalité de cet ordre.

En reprenant donc à la plupart des genres narratifs en vigueur les contradictions qui les fondent, mais en ne faisant fonctionner aucune de ces infractions comme moteur de récit, Buñuel nie leur caractère scandaleux. Il affirme en revanche la compatibilité des contradictoires habituels; on peut être à la fois "dans l'ordre» et "hors-la-loi ", diplomate et crapule, évêque et assassin, père de famille et tortionnaire. Ce n'est pas une affaire, puisqu'il n'y a pas là de quoi faire un film.

\section{Désordre et non-sens}

85 Il ne s'agit donc pas seulement de mettre en cause l'ordre social en montrant un autre fonctionnement social que celui qui est reçu et admis. Il s'agit de contester les principes qui fondent la légitimité de cet ordre, en y contrevenant par tous les moyens, et en leur refusant toute nécessité : la forme de l'infraction aboutit à la négation profonde de la loi.

Cette négation ne concerne pas seulement bien sûr les lois civiles et les représentations acceptées, dont les films de Buñuel récusent la capacité à régler le fonctionnement social et à en rendre compte. Elle atteint, plus profondément, les catégories fondamentales qui nous servent d'ordinaire à parler et à penser, et que la construction contradictoire des films de Buñuel reprend et récuse également. 
87 Par exemple, le principe de causalité. Chez Buñuel comme ailleurs, des liens de cause à conséquence permettent d'ordonner les faits et d'en organiser le récit : ainsi se lient les punitions aux délits, les vengeances aux offenses, les invitations aux repas.

Mais il se passe aussi tout le contraire en une négation récurrente et multiple de tout agencement causal. On l'a vu : ou bien les faits n'ont pas de cause - pas de cause connue en tout cas - (ainsi le complot de l'« auberge rouge » de La voie lactée ou encore la beauté intacte du corps exhumé au début du Fantôme de la liberté ); ou les causes n'ont pas d'effet (crimes impunis, catastrophes sans conséquence, morts après lesquelles on est encore vivant). Les mêmes effets ont des causes différentes (les amis du Charme discret de la bourgeoisie sont libérés par les pressions du ministre aussi bien que par le brigadier sanglant). Les mêmes causes ont des effets différents (ainsi les cartes postales du Fantôme de la liberté tantôt font scandale, tantôt s'intègrent banalement dans le quotidien). Causes et conséquences se trouvent appariées de façon aberrante en des enchaînements qui ne miment les agencements attendus que pour les miner de l'intérieur; erreurs, malentendus, inversions : les fuites sont pour cause de convenance, les invasions pour cause de manœuvres, les duels pour cause de théologie, les scandales pour cause de monuments. De l'absence des enchaînements à leur profusion absurde, tout devient possible.

89 Autre catégorie fondamentale : le temps. Il permet d'habitude de distinguer un avant et un après, d'opposer le passé au présent, de marquer des successions.

90 Tout cela se retrouve chez Buñuel. Et se dissout. La temporalité a bien des allures ordinaires : il y a l'actualité et l'histoire, le présent et le passé, des dates, des durées, des heures, des rendez-vous. Mais tout se mêle : l'histoire d'autrefois fait irruption dans le présent, les siècles se succèdent en désordre (La voie lactée). L'actualité mélange des données anachroniques (dans Le charme discret de la bourgeoisie, par exemple : contestation étudiante et histoire de fantôme, duel et mitraillette, guerre du Vietnam et cavalerie). L'« après " pourrait être " avant ", les successions sont aléatoires : ainsi des rencontres de La voie lactée ou des repas du Charme discret de la bourgeoisie. Les progressions sont absentes, imperceptibles et illusoires, ou contredites : ainsi des voyages sans terme, des repas toujours recommencés, des histoires sans cesse ramenées à leur point de départ. Ni passé, ni futur: le cours du temps n'a rien d'irréversible; les actes ne laissent pas de trace ; il n'y a pas de rides sur les visages et les corps, pas de silence après la mort.

91 Même chose encore pour la hiérarchie. Les films l'affirment par leurs agencements : il y a les utilités et les héros, comme il y a les figurants et les vedettes, les serviteurs et les maîtres, les subalternes et les supérieurs. Dans leur construction même cependant, les films abolissent les privilèges. Les héros se pressent aux premiers rôles et ne se distinguent plus des comparses. Histoires et sujets divers ont droit à la même attention : l'accessoire se mêle au quotidien, le profane au sacré, le signifiant à l'insignifiant.

92 Et dans ce bouleversement général l'identité aussi se trouve finalement récusée. L'histoire des deux préfets sur laquelle se termine le parcours du Fantôme de la liberté est exemplaire de cet aboutissement. À un premier préfet de police se substitue tout à coup un second préfet, puis la substitution fait place à une étonnante confusion. Soudainement et progressivement à la fois, le premier et le second préfet ne font plus qu'un, sans pourtant cesser d'être deux. C'est à la fois le même et l'autre, deux visages et deux présences, une conversation avec des propos qui se répondent mais où, peu à peu, le « nous » fait place à 
un «je » unique, et finalement, un préfet en deux personnes, en une anomalie où se perdent la notion même d'identité et les principes fondamentaux de la logique.

$\mathrm{Au}$ terme du processus, les significations se brouillent ou se dissolvent: les mots et les signes peuvent avoir leur sens ordinaire, mais aussi bien n'importe quel autre - ou aucun. L'affirmation peut être contradictoire: "saint Christophe n'est plus un saint». L'affirmation et la négation peuvent être équivalentes : comme "je » et "nous », "être là » et «n'être pas là » sont parfois employés indifféremment (ainsi dans l'histoire de la fillette «disparue » du Fantôme de la liberté ). Aussi bien les mots parfois ne désignent-ils rien, les fantaisies du dialogue minant le discours sans lui donner de contenu: les mots inventés du Charme discret de la bourgeoisie et du Fantôme de la liberté marquent l'aboutissement d'un procédé déjà à l'œuvre dans le latin sans sous-titre ou l'incompréhensible jargon théologique de La voie lactée.

La faillite de la loi tend alors à détruire la possibilité de signification que fondait cette loi, l'apparence d'intelligibilité donnant sur le refus du sens. Ce refus du sens n'est pas total, mais il est définitif. La dynamique que les films de Buñuel manifestent dans leur pratique contradictoire de la référence aux normes sociales, mon hypothèse est qu'ils la manifestent également dans leur pratique d'élaboration du sens. Ils ne refusent pas toute signification, notamment toute signification critique, et c'est pourquoi, comme on le constate souvent, ils échappent à la gratuité et à l'esthétisme des jeux formels, et ont eu une portée sociale sans commune mesure avec celle des productions dites d'avant-garde. Mais ils refusent toute certitude, toute univocité, toute clôture. Critique à l'égard des cadres que lui fournit l'ordre social dans lequel elle fonctionne, l'entreprise buñuélienne l'est également et continûment à l'égard d'elle-même : à tous les niveaux, tout ce qui peut paraître pouvoir fonctionner comme principe d'unité, tout ce que l'analyse, la réflexion, l'érudition peuvent être tentés de constituer en recours contre la dispersion et l'éclatement du sens se trouve toujours finalement récusé. Le dernier mot reste au paradoxe : on connaît les formules célèbres de l'homme Buñuel, « athée grâce à Dieu » et «fanatiquement anti-fanatique ».

\section{La place du paradoxe}

95 Je voudrais terminer cette étude en évoquant quelques repères qui peuvent permettre de situer dans un contexte plus large le cinéma buñuélien.

À propos de l'œuvre de Buñuel, on parle toujours de « l'Espagne » et du « surréalisme ». Il me paraît intéressant de reformuler ces rapprochements par référence à l'entreprise critique que je me suis efforcée de décrire : aussi bien la tradition espagnole à laquelle peut se rattacher Buñuel (celle du roman picaresque ou de Goya, celle de Cervantes, Gracián ou Calderón, mais aussi celle de la culture populaire et du Carnaval $^{6}$ ) que les textes et les pratiques les plus significatifs de l'attitude surréaliste - qui pourraient également se caractériser par les deux traits complémentaires que j'ai repérés chez Buñuel : d'une part la référence constante à une tradition culturelle et à un système de pensée précisément désignés et visés, d'autre part le refus de l'ordre tel qu'il est défini par cette culture et cette pensée.

97 Mais par cette logique du refus, l'œuvre de Buñuel rejoint également - c'est le constat auquel conduit l'analyse malgré les apparences contraires qu'a installées dans la littérature critique l'exaltation $d u$ mythe de l'«auteur» et le catalogue de ses 
«obsessions »- tout un courant de la pensée et de l'art contemporains qui a repris et développé, sur le plan théorique la critique des valeurs et des systèmes, sur le plan artistique la pratique de l'« œuvre ouverte» et du jeu. On serait tenté par exemple de mettre en face de La voie lactée les recherches et les réflexions modernes sur la notion de vérité qui mettent en évidence son caractère historique et relatif et aboutissent à récuser le concept lui-même ${ }^{7}$. Semblablement, on serait tenté de rapprocher l'obstinée pratique du jeu chez Buñuel d'autres aventures artistiques contemporaines pareillement soustendues par la mise en cause des cadres offerts à la pensée et à l'expression, et de citer, parmi bien d'autres possibles, Borges ou Italo Calvino.

Dans le courant de la culture contemporaine que caractérisent ainsi critique et « inacceptation », l'originalité de Buñuel est double.

Elle est d'abord - constatation bien triviale - dans sa réussite : rares sans doute sont ceux qui ont su poursuivre aussi longtemps, aussi complètement et aussi parfaitement qu'il l'a fait la réalisation de ce projet périlleux entre tous qui ne se satisfait ni des cadres offerts à la parole ni du silence, ni du néant ni du possible; l'équilibre est fragile à établir et à renouveler pour éviter que la communication s'interrompe, pour éviter qu'en fonctionnant elle sanctionne ce qu'on voulait refuser. Nombreux sont les joueurs qui, au terme de leur refus, finissent par jouer tout seuls, ou presque ; nombreux aussi sont ceux - le surréalisme en a compté, et stigmatisé, plus d'un - qui d'une façon ou d'une autre reviennent à l'ordre. Ce qui fait l'intérêt de l'entreprise buñuélienne c'est d'avoir su éviter l'un et l'autre écueil et poursuivre indéfiniment la subversion initialement choisie.

L'autre originalité de Buñuel c'est d'avoir su développer cette pratique du jeu dans un art que son fonctionnement social défend sans doute plus que d'autres des entreprises de désordre, et de l'avoir développée à un degré à peine imaginable puisqu'il n'est guère d'aspects du film qu'ait épargnés sa subversion. Dans le cinéma même, c'est sans doute la constance de cette subversion, la richesse et l'intelligence de l'invention par laquelle elle s'opère qui peuvent le mieux permettre de caractériser et de situer l'« œuvre » de Buñuel, tandis que la prise en compte du jeu peut conduire à rapprocher sa pratique et ses films de ceux d'autres cinéastes dont l'allure semble de prime abord fort différente.

On pourrait citer par exemple le Godard de Pierrot le fou qui opère une subversion du récit dont les points communs sont nombreux avec la subversion buñuélienne: il part d'un roman policier banal dont il reprend la plupart des données (les personnages principaux, la trame narrative, divers épisodes essentiels, des pans entiers du dialogue, de nombreux détails) mais pour en bouleverser l'économie générale par des procédés analogues aux procédés buñuéliens (manques, excès, déplacements en tous genres) si bien que le film se construit contre le récit canonique auquel il fait référence et dont il ne cesse de brouiller et d'ouvrir les significations.

Dans une autre direction - ou la même? - on pourrait aussi citer le Wajda de L'homme de marbre qui met en œuvre une critique de la vérité dont les rencontres sont nombreuses avec celle qu'opèrent notamment Le charme discret de la bourgeoisie d'une part, La voie lactée de l'autre. Révélation de la mise en scène et des procédures de construction et d'exclusion qui constituent la vérité comme telle, choc de vérités contradictoires qui s'affrontent en même temps ou d'un temps à l'autre, mise en cause à la fois subtile et radicale du discours du film lui-même impliqué comme les autres discours dans le procès de la vérité qu'il conduit ; c'est, à travers un fonctionnement historiquement situé, la notion même de vérité que Wadja, comme Buñuel, interroge et récuse. 
Ce ne sont là que des exemples - parmi d'autres possibles - de rencontres que justifie le repérage, chez Godard ou chez Wajda, de cette même attitude de refus, d'« inacceptation» ou, comme je l'ai appelé, de jeu, qui me semble caractériser plus qu'aucun autre trait les films de Buñuel.

\section{BIBLIOGRAPHIE}

RÉFÉRENCES DES FILMS CITÉS

\section{Films de Luis Buñuel}

Un chien andalou, France, 1929.

L'âge d'or, France, 1930.

Los Olvidados, Mexique, 1950.

L'enjôleuse (El bruto), Mexique, 1952.

Les hauts de Hurlevent (Abismos de pasión), Mexique, 1953.

Nazarin, Mexique, 1958.

Viridiana, Espagne, 1961.

L'ange exterminateur (El angel exterminador), Mexique, 1962.

Le journal d'une femme de chambre, France, 1963.

Belle de jour, France, 1966.

La voie lactée, France, 1969.

Le charme discret de la bourgeoisie, France, 1972.

Le fantôme de la liberté, France, 1974.

Cet obscur objet du désir, France, 1977.

\section{Autres films}

Alphaville, Jean-Luc Godard. France, 1965.

Le gendarme de Saint-Tropez, Jean Girault, France, 1964.

Le grand blond avec une chaussure noire, Yves Robert, France, 1972.

L'homme de marbre, Andrzej Wajda, Pologne, 1977.

Pierrot le fou, Jean-Luc Godard, France, 1965. 


\section{NOTES}

1. Ce texte s'appuie sur les recherches présentées dans ma thèse pour le doctorat d'État (Luis Buñuel. Le jeu et la loi, Université Paris X-Nanterre, 1986). On pourra se reporter à cet ouvrage pour des analyses et des références plus complètes.

2. Pour les références des films, cf. la liste alphabétique placée à la fin du texte.

3. Cf. Le Cinématographe, 10, nov.-déc. 1974, p. 11 ; et P. Bonitzer, "La table de dissection (Le fantôme de la liberté)", Les Cahiers du Cinéma, 253, oct.-nov. 1974, p. 37-38. Pour l'ensemble des références aux critiques, qu'il me semble ici inutile de préciser chaque fois dans le détail, cf. mon texte cité à la note 1, Luis Buñuel. Le jeu et la loi.

4. M. Aymé, La convention Belzébir, Paris, Gallimard, 1967.

5. Pour ce point, cf. T. Todorov, «La grammaire du récit: le Décaméron », in Poétique de la prose, Paris, Seuil, 1978, p. 50-51.

6. La pratique buñuélienne du jeu rencontre très précisément la tradition de la culture populaire telle que l'a décrite Bakhtine, toute cette culture du Carnaval qui met le monde "à l'envers", «s'affranchit » de la vérité " dominante ", « abolit » « tous les rapports hiérarchiques, privilèges, règles et tabous ». Les mêmes traits constitutifs de la rupture et du jeu se retrouvent dans les deux cas, qu'il s'agisse de la logique du renversement, des "permutations constantes du haut et du bas", "de la face et du derrière", des «formes [...] diverses des parodies et travestissements », de la libération à l'égard des « liens de la logique ou de la signification », ou de la négation de l'achèvement et de l'unité. Cf. M. Bakhtine, L'œuvre de François Rabelais et la culture populaire au Moyen Âge et sous la Renaissance, Paris, Gallimard, 1970 (notamment p. 18, 19, 370, 429).

7. Sur ce point, cf. par exemple M. Detienne, Les maîtres de vérité dans la Grèce archaïque, Paris, Maspero, 1967 ; M. Foucault, L'ordre du discours, Paris, Gallimard, 1971 ; ou T. S. Kuhn, La structure des révolutions scientifiques, Paris, Flammarion, 1972. 